\title{
Asymptotically Optimal Box Packing Theorems
}

\author{
Michael Reid \\ Department of Mathematics \\ University of Central Florida, Orlando, FL 32816 \\ reid@math.ucf .edu
}

Submitted: Dec 29, 2007; Accepted: Jun 3, 2008; Published: Jun 6, 2008

Mathematics Subject Classifications: 05C15, (52C17 05B50)

\begin{abstract}
Given a protoset of $d$-dimensional polyominoes, we ask which boxes can be packed by the protoset. In some cases, it may be too difficult to give a complete answer to this question, so we ask the easier question about determining all sufficiently large boxes that can be packed. (We say that a box is "sufficiently large" if all edge lengths are $\geq C$ for some large $C$.) We give numerous examples (mostly 2-dimensional) where we can answer this easier question. The various techniques involved are: checkerboard-type colorings/numberings (tile homology), the boundary word method of Conway and Lagarias (tile homotopy), ad hoc geometric arguments, and a very nice theorem of Barnes. Barnes' Theorem asserts that all necessary conditions for a box to be packable can be given in a certain form, and these conditions are also sufficient for large boxes.

Barnes' Theorem has not received the appreciation it deserves. We give a new, purely combinatorial proof of this important result. (Barnes' original proof uses techniques of algebraic geometry.) In the special case that all the prototiles are boxes themselves, we show how to determine all sufficiently large boxes that they pack. We prove a theorem based on Barnes' result that reduces this to a straightforward calculation.
\end{abstract}

\section{Introduction}

A $d$-dimensional polyomino is a union of unit $d$-dimensional cubes such that the vertices of each are lattice points. It is common to require that they have connected interior, but we do not impose that condition here. A protoset is a collection of prototiles, each of which we have an unlimited supply. Given a finite polyomino region, we ask if the region can be packed by the protoset, which means that the region is the union of polyominoes that intersect at most along their boundaries, and such that each is congruent to one of our prototiles. In general, this is a difficult problem (see [17]), and we concentrate on the special case when the region to be packed is a box (rectangular parallelepiped). There are several reasons for interest in this special case. Firstly, boxes 
are the most basic type of finite regions to consider. Secondly, this problem has already attracted some attention; for example, see $[1,2,3,5,7,8,9,11,12,14,15,16,20,22$, 24]. Thirdly, we use two (known) structure theorems, which we describe in section 3 below. We include a new proof of one of them, and prove a useful new result (Theorem 3.8) based upon them.

Given a protoset, we can ask for a complete determination of all boxes that it packs. This may be difficult to attain, even for relatively simple protosets. See Example 4.2 below to get an idea of how complex this determination can be. If we cannot find all boxes that can be packed, we may ask instead for all "sufficiently large" boxes that can be packed by the protoset. That is, for $C \gg 0$, determine all $a_{1} \times \cdots \times a_{d}$ boxes with each $a_{i} \geq C$ that can be packed. This is often more tractable, even in an extreme case like Example 4.2 below. Some results of this type have been given in $[10,14]$.

\section{Klarner systems}

In this section we develop the concept of Klarner systems, which was introduced in [22] as a convenient framework in which to consider these packing problems.

Definition 2.1. Let $d$ be a positive integer. A $d$-dimensional Klarner system, is a subset $S$ of $\mathbb{N}^{d}$ such that if both $\left(a_{1}, a_{2}, \ldots, a_{d}\right)$ and $\left(a_{1}, a_{2}, \ldots, a_{i-1}, a_{i}^{\prime}, a_{i+1}, \ldots, a_{d}\right)$ are in $S$, then so is $\left(a_{1}, a_{2}, \ldots, a_{i-1}, a_{i}+a_{i}^{\prime}, a_{i+1}, \ldots, a_{d}\right)$.

Our motivation is provided by the following example.

Example 2.2. Let $\mathcal{T}$ be any protoset of translation-only $d$-dimensional polyominoes, and let $S=\left\{\left(a_{1}, a_{2}, \ldots, a_{d}\right) \in \mathbb{N}^{d} \mid \mathcal{T}\right.$ packs an $a_{1} \times a_{2} \times \cdots \times a_{d}$ box $\}$. Then $S$ is a $d$-dimensional Klarner system. Indeed, if $\mathcal{T}$ packs an $a_{1} \times \cdots \times a_{d}$ box and also an $a_{1} \times \cdots \times a_{i}^{\prime} \times \cdots \times a_{d}$ box, then these two boxes can be juxtaposed (using only translation) to give a packing of an $a_{1} \times \cdots \times\left(a_{i}+a_{i}^{\prime}\right) \times \cdots \times a_{d}$ box.

The condition that the prototiles be translation-only may seem unusual. Oftentimes, we will want to be able to use a prototile in any orientation. To do this, we include every orientation of the tile in the protoset. This shows that translation-only protosets are more general than protosets where we may rotate and reflect the tiles, which is our reason for considering them.

In view of this motivating example, we will refer to elements of a Klarner system as "boxes". This will be useful to distinguish them from other tuples of integers that we will have later.

We also note that boxes can pack larger boxes in more complex ways than simply juxtaposing two smaller boxes to made a larger box, and repeating this process. For example, a $3 \times 3$ square and four $1 \times 4$ rectangles (used in both orientations) can pack a $5 \times 5$ square, but cannot do so with a line of cleavage.

Lemma 2.3. (a) A non-empty intersection of Klarner systems is a Klarner system.

(b) An increasing union of a sequence of Klarner systems is a Klarner system.

Proof. Clear. 
Definition 2.4. Let $\mathcal{U}$ be a subset of $\mathbb{N}^{d}$. The Klarner system generated by $\mathcal{U}$ is the smallest Klarner system containing $\mathcal{U}$; i.e. the intersection of all Klarner systems that contain $\mathcal{U}$.

Example 2.5. The Klarner system generated by $\{(3,3),(1,4),(4,1)\}$ does not contain the box $(5,5)$ although a $5 \times 5$ square can be packed by the corresponding rectangles.

Definition 2.6. A prime of a Klarner system $S$ is an element of $S$ that cannot be written as $\left(a_{1}, \ldots, a_{i-1}, a_{i}+a_{i}^{\prime}, a_{i+1}, \ldots, a_{d}\right)$ where both $\left(a_{1}, \ldots, a_{d}\right)$ and $\left(a_{1}, \ldots, a_{i}^{\prime}, \ldots, a_{d}\right)$ are in $S$.

In connection to the motivating example above, a prime corresponds to a box that can be tiled by the protoset, but cannot be tiled with a plane of cleavage.

The next two results, which are from [22], are easy, so we omit their proofs.

Lemma 2.7. An element $\left(a_{1}, a_{2}, \ldots, a_{d}\right)$ of a Klarner system $S$ is prime if and only if $S \backslash\left\{\left(a_{1}, a_{2}, \ldots, a_{d}\right)\right\}$ is also a Klarner system.

Corollary 2.8. The unique minimal generating set of a Klarner system is its set of primes.

\section{Fundamental Structure Theorems}

In this section, we give the two fundamental theorems. Since they were not originally stated in the context of Klarner systems, we have translated them into that context. In view of the motivating example above, the connection to the original context should be clear.

The first fundamental theorem is due to Klarner and Göbel.

Theorem 3.1. (Klarner and Göbel) A d-dimensional Klarner system has only finitely many primes.

This theorem was first given in [11], but the proof had an error in dimensions $d \geq 3$. The proof was later repaired by Klarner in an unpublished technical report [13]. Other proofs have been given by de Bruijn and Klarner [6], Bodini [2], and by the current author [22]. The theorem shows that a Klarner system can be described by giving its finite set of primes. However, it is not clear if this is the most convenient description. Bodini [2] discusses the problem of determining if a given box can be packed by a given set of prototile boxes.

Definitions 3.2. A restriction is simply a $d$-tuple $\left[r_{1}, r_{2}, \ldots, r_{d}\right]$ of non-negative integers. We will use brackets for restrictions to distinguish them from boxes. We say that a box $\left(a_{1}, a_{2}, \ldots, a_{n}\right)$ satisfies the restriction $\left[r_{1}, r_{2}, \ldots, r_{d}\right]$ if $a_{i}$ is a multiple of $r_{i}$ for some index $1 \leq i \leq d$. We say that a Klarner system satisfies a restriction if all of its boxes satisfy the restriction. We also adopt the convention that 0 divides $b$ if and only if $b=0$, so that " $a$ divides $b$ " is synonymous with " $b$ is a multiple of $a$ " in all cases. We say the restriction $\left[r_{1}, \ldots, r_{d}\right]$ divides the restriction $\left[s_{1}, \ldots, s_{d}\right]$ if $r_{i} \mid s_{i}$ for 
all $i$. A restriction $\left[r_{1}, \ldots, r_{d}\right]$ is called primary if each $r_{i}$ is either a prime power or 0 . We will call a restriction $\left[q_{1}, \ldots, q_{d}\right]$ a primary divisor of the restriction $\left[r_{1}, \ldots, r_{d}\right]$ if it is primary, and it divides $\left[r_{1}, \ldots, r_{d}\right]$, and $q_{i}=0$ if $r_{i}=0$. This last condition ensures that every restriction has only finitely many primary divisors.

The second fundamental theorem is due to Barnes [1].

Theorem 3.3. (Barnes) Let $S$ be a $d$-dimensional Klarner system. There is a finite set of restrictions $\mathcal{R}$ and a constant $C>0$ such that

(a) Every box in $S$ satisfies every restriction in $\mathcal{R}$, and

(b) If $a_{1}, a_{2}, \ldots, a_{d} \geq C$, then the box $\left(a_{1}, a_{2}, \ldots, a_{d}\right)$ is in $S$ if and only if it satisfies every restriction in $\mathcal{R}$.

The "only if" part of (b) is redundant, but it allows for a necessary and sufficient condition. We will say that a finite set of restrictions, $\mathcal{R}$, characterizes a Klarner system, if it satisfies the conditions of the theorem for some choice of $C$.

Barnes did not state his theorem in terms of Klarner systems, so the above is a translation of his original statement. He also stated it only for finite protosets, so he was apparently unaware of the theorem of Klarner and Göbel. Barnes' Theorem does not seem to be adequately appreciated, which is part of our motivation for presenting it here. His original proof was based on developing some concepts in classical algebraic geometry. We give below a combinatorial proof of this important theorem. His statement also included more than we have here; it included information about packing with general weights, which is not of concern to us here.

A special case of Barnes' Theorem, in which the tiles may be rotated, was given earlier by Katona and Szász [10, Thm. 2 and 3], with a rather cumbersome statement.

Determining the smallest value of $C$ in the theorem is an interesting issue, but not one that we will address here. Even in the 1-dimensional case, it is interesting; in this case, it is essentially the Frobenius problem. (See [19] for more about various aspects of the Frobenius problem.) In the 1-dimensional case, the necessary restrictions for a box to be packable are also sufficient, with finitely many exceptions. In the higher dimensional case, there may be infinitely many exceptions, as we will see below.

The set of restrictions in the theorem is not uniquely determined. If $\left[r_{1}, r_{2}, \ldots, r_{d}\right]$ is a restriction, then so is any restriction that divides $\left[r_{1}, r_{2}, \ldots, r_{d}\right]$. Therefore it makes sense to only consider restrictions that are maximal with respect to division. Even then, the set of restrictions is not uniquely determined. One way to achieve uniqueness is to replace each restriction by its set of primary divisors and then choose maximal elements from this collection. In general, this greatly increases the number of restrictions. It is unclear if it makes the set of restrictions more "natural" in any sense, so we will not pursue it here, although it will be useful in the proof of Theorem 3.8 below.

Proof of Theorem 3.3. First we show that (a) follows from (b). Suppose $\left(a_{1}, \ldots, a_{d}\right)$ is a box in $S$. Let $N$ be a prime larger than $C$ that does not divide any non-zero coordinate of any restriction in $\mathcal{R}$. The box $\left(N a_{1}, \ldots, N a_{d}\right)$ is in $S$, so by (b), it satisfies every restriction in $\mathcal{R}$. Thus, if $\left[r_{1}, \ldots, r_{d}\right] \in \mathcal{R}$, then there is an index $i$ such 
that $r_{i} \mid N a_{i}$. Now $r_{i} \neq 0$ is relatively prime to $N$, so $r_{i} \mid a_{i}$. Therefore the original box $\left(a_{1}, \ldots, a_{d}\right)$ also satisfies the restriction, so (a) holds.

Now we prove (b) by induction on the dimension $d$. Suppose that $d=1$. If $S=\varnothing$, then (b) is clear: take $\mathcal{R}=\{[0]\}$ and $C=1$. If $S$ is non-empty, let $g$ be the greatest common divisor of elements of $S$. We may choose a finite subset $a_{1}<a_{2}<\cdots<a_{k}$ of $S$ such that $g=\operatorname{GCD}\left(a_{1}, \ldots, a_{k}\right)$. Every box in $S$ satisfies the restriction $[g]$. Conversely, if $n$ is divisible by $g$, it may be expressed as $a_{1} x_{1}+a_{2} x_{2}+\cdots+a_{k} x_{k}$ for some integers $x_{1}, \ldots, x_{k}$. Now also suppose that $n \geq\left(a_{1}-1\right)\left(a_{2}+a_{3}+\cdots+a_{k}\right)$. For each $i=2,3, \ldots, k$, we may replace $x_{i}$ by $x_{i}^{\prime}=x_{i}-t a_{1}$ and $x_{1}$ by $x_{1}+t a_{i}$, for some integer $t$ so that $0 \leq x_{i}^{\prime}<$ $a_{1}$. Then $a_{1} x_{1}=n-\left(a_{2} x_{2}+a_{3} x_{3}+\cdots+a_{k} x_{k}\right) \geq n-\left(a_{1}-1\right)\left(a_{2}+a_{3}+\cdots+a_{k}\right) \geq 0$, so all the $x_{i}$ 's are non-negative, whence $n \in S$. Therefore (b) holds with $\mathcal{R}=\{[g]\}$ and $C=\left(a_{1}-1\right)\left(a_{2}+a_{3}+\cdots+a_{k}\right)$.

Now suppose that (b) holds for all $(d-1)$-dimensional Klarner systems and let $S$ be a $d$-dimensional Klarner system. For a positive integer $n$, let

$$
S(n)=\left\{\left(a_{1}, a_{2}, \ldots, a_{d-1}\right) \in \mathbb{N}^{d-1} \mid\left(a_{1}, a_{2}, \ldots, a_{d-1}, n\right) \in S\right\},
$$

which is a $(d-1)$-dimensional Klarner system. We also have $S\left(n_{1}\right) \cap S\left(n_{2}\right) \subseteq S\left(n_{1}+n_{2}\right)$, and then $S(n) \subseteq S(k n)$ follows from this by induction on $k$.

Now we have an increasing chain $S(1 !) \subseteq S(2 !) \subseteq S(3 !) \subseteq \cdots$, which stabilizes, as every Klarner system has a finite number of generators. Thus $\bigcup_{k} S(k !)=S(n !)$ for some $n$. Since $S(k) \subseteq S(k !)$, this shows that there is a positive integer $m$ such that $S(m)$ is maximal. For the remainder of the proof, $m$ will denote such an integer. For each $0<n \leq m$, we have an increasing chain $S(n) \subseteq S(n+m) \subseteq S(n+2 m) \subseteq \cdots$, which also stabilizes.

Let $T(n)=\bigcup_{t} S(n+t m)$, so that $T(n)=S(n+t m)$ for sufficiently large $t$. Of course $T(n)$ depends only on the remainder of $n$ modulo $m$. We claim that

$$
T\left(n_{1}\right) \cap T\left(n_{2}\right) \cap \cdots \cap T\left(n_{r}\right) \subseteq T\left(\operatorname{GCD}\left(n_{1}, n_{2}, \ldots, n_{r}\right)\right) .
$$

It suffices to prove (3.4) for $r=2$; the general case follows by induction on $r$. We may write $\operatorname{GCD}\left(n_{1}, n_{2}\right)=k_{1} n_{1}+k_{2} n_{2}$ for some integers $k_{1}, k_{2}$. Choose positive integers $k_{1}^{\prime}, k_{2}^{\prime}$ such that $k_{i}^{\prime} \equiv k_{i} \bmod m$. For large $t$, we have $T\left(n_{i}\right)=S\left(n_{i}+t m\right) \subseteq S\left(k_{i}^{\prime}\left(n_{i}+t m\right)\right)$, so that $T\left(n_{1}\right) \cap T\left(n_{2}\right) \subseteq S\left(k_{1}^{\prime}\left(n_{1}+t m\right)\right) \cap S\left(k_{2}^{\prime}\left(n_{2}+t m\right)\right) \subseteq S\left(k_{1}^{\prime} n_{1}+k_{2}^{\prime} n_{2}+\left(k_{1}^{\prime}+k_{2}^{\prime}\right) t m\right) \subseteq$ $T\left(k_{1}^{\prime} n_{1}+k_{2}^{\prime} n_{2}\right)=T\left(k_{1} n_{1}+k_{2} n_{2}\right)$, which proves the claim.

As a special case of $(3.4)$, we note that $T(n)=T(n) \cap T(m) \subseteq T(\operatorname{GCD}(m, n))$.

For each divisor $n$ of $m$, fix a finite set, $\mathcal{R}(n)$, of restrictions that characterizes the $(d-1)$-dimensional Klarner system $T(n)$. Let $\mathcal{R}$ be the union of the following sets of restrictions:

$$
\mathcal{R}_{0}=\left\{\left[r_{1}, \ldots, r_{d-1}, 0\right] \mid\left[r_{1}, \ldots, r_{d-1}\right] \in \mathcal{R}(m)\right\},
$$

and for each prime power $p^{f}$ that divides $m$, let $p^{e}$ be the largest power of $p$ dividing $m$, and set

$$
\mathcal{R}_{p^{f}}=\left\{\left[r_{1}, \ldots, r_{d-1}, p^{f}\right] \mid\left[r_{1}, \ldots, r_{d-1}\right] \in \mathcal{R}\left(m / p^{e-f+1}\right)\right\}
$$


To complete the induction, we will show that $\mathcal{R}$ characterizes $S$.

First we show that every box in $S$ satisfies every restriction in $\mathcal{R}$. Suppose that $\left(a_{1}, \ldots, a_{d}\right)$ is a box in $S$. Then $\left(a_{1}, \ldots, a_{d-1}\right) \in S\left(a_{d}\right) \subseteq S(m)=T(m)$, so it satisfies every restriction in $\mathcal{R}(m)$. Thus $\left(a_{1}, \ldots, a_{d}\right)$ satisfies every restriction in $\mathcal{R}_{0}$. Let $p^{f}$ be a prime power that divides $m$. If $p^{f} \mid a_{d}$, then $\left(a_{1}, \ldots, a_{d}\right)$ satisfies every restriction in $\mathcal{R}_{p^{f}}$. Otherwise, $\operatorname{GCD}\left(a_{d}, m\right)$ divides $m / p^{e-f+1}$, so we have $\left(a_{1}, \ldots, a_{d-1}\right) \in$ $S\left(a_{d}\right) \subseteq T\left(a_{d}\right) \subseteq T\left(\operatorname{GCD}\left(a_{d}, m\right)\right) \subseteq T\left(m / p^{e-f+1}\right)$, so it satisfies every restriction in $\mathcal{R}\left(m / p^{e-f+1}\right)$. Therefore $\left(a_{1}, \ldots, a_{d}\right)$ satisfies every restriction in $\mathcal{R}_{p^{f}}$, which shows that $S$ satisfies every restriction in $\mathcal{R}$.

Now we show that $S$ contains every sufficiently large box that satisfies all restrictions in $\mathcal{R}$. Choose $C$ large enough so that $S(k)=T(k)$ for all $k \geq C$, and such that for every $n \mid m$, if $a_{1}, \ldots, a_{d-1} \geq C$ and the box $\left(a_{1}, \ldots, a_{d-1}\right)$ satisfies every restriction in $\mathcal{R}(n)$, then it is in $T(n)$.

Suppose that $a_{1}, \ldots, a_{d} \geq C$ and the box $\left(a_{1}, \ldots, a_{d}\right)$ satisfies every restriction in $\mathcal{R}$. For a prime $p$ that divides $m$, let $p^{e}$ be the largest power of $p$ dividing $m$, and let $p^{f}$ be the largest power of $p$ that divides $\operatorname{GCD}\left(a_{d}, m\right)$. We claim that the box $\left(a_{1}, \ldots, a_{d-1}\right)$ is in $T\left(m / p^{e-f}\right)$. If $f<e$, then $p^{f+1}$ divides $m$ but does not divide $a_{d}$. Since $\left(a_{1}, \ldots, a_{d}\right)$ satisfies every restriction in $\mathcal{R}_{p^{f+1}}$, it follows that the box $\left(a_{1}, \ldots, a_{d-1}\right)$ satisfies every restriction in $\mathcal{R}\left(m / p^{e-f}\right)$. Therefore, $\left(a_{1}, \ldots, a_{d-1}\right) \in T\left(m / p^{e-f}\right)$, because $a_{1}, \ldots, a_{d-1} \geq C$, so the claim holds in this case.

If $f=e$, the box $\left(a_{1}, \ldots, a_{d}\right)$ satisfies every restriction in $\mathcal{R}_{0}$, so it follows that $\left(a_{1}, \ldots, a_{d-1}\right)$ satisfies every restriction in $\mathcal{R}(m)=\mathcal{R}\left(m / p^{e-f}\right)$, and as before, we have $\left(a_{1}, \ldots, a_{d-1}\right) \in T\left(m / p^{e-f}\right)$. This proves the claim in all cases.

Finally, write $n=\operatorname{GCD}\left(a_{d}, m\right)=p_{1}^{f_{1}} \cdots p_{r}^{f_{r}}$, where we include every prime divisor of $m$ (so some exponents may be 0 ). From the claim, we have $\left(a_{1}, \ldots, a_{d-1}\right) \in$ $T\left(m / p_{i}^{e_{i}-f_{i}}\right)$ for each $i$, so by (3.4) above, we have $\left(a_{1}, \ldots, a_{d-1}\right) \in \bigcap_{i} T\left(m / p_{i}^{e_{i}-f_{i}}\right) \subseteq$ $T\left(\operatorname{GCD}\left(\left\{m / p_{i}^{e_{i}-f_{i}}\right\}\right)\right)=T(n) \subseteq T\left(a_{d}\right)$. Since $a_{d} \geq C$, we have $T\left(a_{d}\right)=S\left(a_{d}\right)$, which shows that the box $\left(a_{1}, \ldots, a_{d}\right)$ is in $S$.

Remark 3.5. In the proof of Theorem 3.3, we used Theorem 3.1 during the induction step. However, we used it only for $(d-1)$-dimensional Klarner systems. The beginning of the induction step follows along the same lines as the inductive proof of Theorem 3.1 given in [22], so one may even prove both theorems at once, inducting on the dimension. In comparison, the proof of Theorem 3.3 requires more analysis. This is curious in light of the fact that the set of primes determines the Klarner system completely, but the set of restrictions only determines its "sufficiently large" boxes.

Let $\mathcal{U} \subseteq \mathbb{N}^{d}$ be a set of boxes, and $\mathcal{R}$ a set of restrictions. If every box in $\mathcal{U}$ satisfies every restriction in $\mathcal{R}$, then the same holds for every box in the Klarner system generated by $\mathcal{U}$. Indeed, the set of boxes that satisfies every restriction in $\mathcal{R}$ is a Klarner system, and it contains $\mathcal{U}$. Barnes has shown that restrictions are preserved even under more complex box-packing, which is the content of the next theorem.

Theorem 3.6. (Barnes) Suppose that every box in the set $S$ satisfies the restriction 
$\left[r_{1}, \ldots, r_{d}\right]$. If a box can be tiled by the boxes in $S$ by translation only, then this box also satisfies the restriction $\left[r_{1}, \ldots, r_{d}\right]$.

This theorem is the discrete analogue of the main theorem treated by Wagon [23]. If $S_{1}$ and $S_{2}$ are two $d$-dimensional Klarner systems, let $S_{1}+S_{2}$ denote the Klarner system generated by $S_{1} \cup S_{2}$. Based on Theorem 3.3, we can understand how restrictions behave under this operation. First we have an easy lemma.

Lemma 3.7. Suppose the Klarner system $S$ is characterized by the finite set of restrictions $\mathcal{R}$. If $\left[q_{1}, q_{2}, \ldots, q_{d}\right]$ is a primary restriction that every box of $S$ satisfies, then $\left[q_{1}, q_{2}, \ldots, q_{d}\right]$ divides some restriction in $\mathcal{R}$.

Proof. We prove the contrapositive. Suppose that $\left[q_{1}, q_{2}, \ldots, q_{d}\right]$ does not divide any restriction in $\mathcal{R}$. Then, for all $\left[r_{1}, \ldots, r_{d}\right] \in \mathcal{R}$, there is an index $i$ such that $q_{i} \nmid r_{i}$. Put $\mathcal{R}^{(i)}=\left\{\left[r_{1}, \ldots, r_{d}\right] \mid q_{i} \nmid r_{i}\right\}$, and let $a_{i}=\operatorname{LCM}\left(\left\{r_{i} \mid\left[r_{1}, \ldots, r_{d}\right] \in \mathcal{R}^{(i)}\right\}\right)$. Since $q_{i}$ is a prime power or 0 , and $a_{i}$ is the least common multiple of non-zero integers, none of which is divisible by $q_{i}$, it follows that $a_{i}$ is also not a multiple of $q_{i}$. This holds for all $i$, so the box $\left(a_{1}, \ldots, a_{d}\right)$ does not satisfy the restriction $\left[q_{1}, \ldots, q_{d}\right]$. On the other hand, $\left(a_{1}, \ldots, a_{d}\right)$ satisfies all the restrictions in each $\mathcal{R}^{(i)}$, and since $\mathcal{R}^{(1)} \cup \mathcal{R}^{(2)} \cup \ldots \cup \mathcal{R}^{(d)}=\mathcal{R}$, it satisfies all the restrictions in $\mathcal{R}$. The same holds for $\left(N a_{1}, \ldots, N a_{d}\right)$, so if $N$ is sufficiently large, the box $\left(N a_{1}, \ldots, N a_{d}\right)$ is in the Klarner system $S$. If $N$ is also chosen to be relatively prime to all the non-zero coordinates of $\left[q_{1}, \ldots, q_{d}\right]$, then $\left(N a_{1}, \ldots, N a_{d}\right)$ does not satisfy this restriction. This proves the contrapositive.

If $\left[r_{1}, \ldots, r_{d}\right]$ and $\left[s_{1}, \ldots, s_{d}\right]$ are two restrictions, let $\left[r_{1}, \ldots, r_{d}\right] \vee\left[s_{1}, \ldots, s_{d}\right]$ denote $\left[\operatorname{GCD}\left(r_{1}, s_{1}\right), \ldots, \operatorname{GCD}\left(r_{d}, s_{d}\right)\right]$, where, as a matter of convention, we put $\operatorname{GCD}(0, a)=$ $a=\operatorname{GCD}(a, 0)$ for all non-negative integers $a$.

Theorem 3.8. Suppose that $S_{1}$ and $S_{2}$ are $d$-dimensional Klarner systems, characterized by the finite sets of restrictions $\mathcal{R}_{1}$ and $\mathcal{R}_{2}$ respectively. The Klarner system $S_{1}+S_{2}$ is characterized by the finite set of restrictions

$$
\mathcal{R}_{1} \vee \mathcal{R}_{2}=\left\{\left[r_{1}, \ldots, r_{d}\right] \vee\left[s_{1}, \ldots, s_{d}\right] \mid\left[r_{1}, \ldots, r_{d}\right] \in \mathcal{R}_{1},\left[s_{1}, \ldots, s_{d}\right] \in \mathcal{R}_{2}\right\} .
$$

Proof. It is clear that every box in $S_{1} \cup S_{2}$ satisfies every restriction in $\mathcal{R}_{1} \vee \mathcal{R}_{2}$, and therefore so does every box in the Klarner system $S_{1}+S_{2}$. By Theorem 3.3, $S_{1}+S_{2}$ is characterized by a finite set of restrictions, $\mathcal{R}$, and we may also assume that every restriction in $\mathcal{R}$ is primary. Since $S_{1} \subseteq S_{1}+S_{2}$, every box in $S_{1}$ satisfies every restriction $\left[q_{1}, \ldots, q_{d}\right]$ in $\mathcal{R}$. From the lemma, $\left[q_{1}, \ldots, q_{d}\right]$ divides some restriction $\left[r_{1}, \ldots, r_{d}\right]$ in $\mathcal{R}_{1}$, and similarly, it divides some $\left[s_{1}, \ldots, s_{d}\right]$ in $\mathcal{R}_{2}$. Thus $\left[q_{1}, \ldots, q_{d}\right]$ divides the restriction $\left[r_{1}, \ldots, r_{d}\right] \vee\left[s_{1}, \ldots, s_{d}\right]$ in $\mathcal{R}_{1} \vee \mathcal{R}_{2}$. This shows that every restriction in $\mathcal{R}$ divides some restriction in $\mathcal{R}_{1} \vee \mathcal{R}_{2}$. Therefore, if $\left(a_{1}, \ldots, a_{d}\right)$ is a sufficiently large box that satisfies all the restrictions in $\mathcal{R}_{1} \vee \mathcal{R}_{2}$, then it satisfies every restriction in $\mathcal{R}$, so it is in the Klarner system $S_{1}+S_{2}$. This proves the theorem.

Example 3.9. A problem from the Putnam Competition in 1991 asks 
Does there exist a real number $L$ such that, if $m$ and $n$ are integers greater than $L$, then an $m \times n$ rectangle may be expressed as a union of $4 \times 6$ and $5 \times 7$ rectangles, any two of which intersect at most along their boundaries?

The rectangles may be rotated, so we are concerned with the Klarner system generated by $(4,6),(6,4),(5,7)$ and $(7,5)$. The Klarner system generated by $(4,6)$ is simply $\{(4 m, 6 n)\}$, which is characterized by the restrictions $\{[4,0],[0,6]\}$. Likewise, the Klarner system generated by $(5,7)$ is characterized by $\{[5,0],[0,7]\}$. Therefore, by Theorem 3.8 , the Klarner system generated by $(4,6)$ and $(5,7)$ is characterized by the set of restrictions

$$
\{[4,0] \vee[5,0],[4,0] \vee[0,7],[0,6] \vee[5,0],[0,6] \vee[0,7]\}=\{[1,0],[4,7],[5,6],[0,1]\}
$$

Any restriction with a coordinate equal to 1 is automatically satisfied by every box, so these can be removed. Thus the Klarner system generated by $(4,6)$ and $(5,7)$ is characterized by $\{[4,7],[5,6]\}$. By interchanging the coordinates, we see that the Klarner system generated by $(6,4)$ and $(7,5)$ is characterized by $\{[7,4],[6,5]\}$. Apply Theorem 3.8 to these two Klarner systems; we find that the Klarner system generated by $(4,6),(6,4)$, $(5,7)$ and $(7,5)$ is characterized by $\{[4,7] \vee[7,4],[4,7] \vee[6,5],[5,6] \vee[7,4],[5,6] \vee[6,5]\}$ $=\{[1,1],[2,1],[1,2],[1,1]\}$. Since these restrictions are all irrelevant, the Klarner system is characterized by the empty set of restrictions. This proves that the answer to the Putnam problem is "yes".

This illustrates the power of Theorem 3.8; it reduces this type of problem to a simple calculation, as above. One can show, by an elementary, but somewhat tedious computation, that the Klarner system generated by $(4,6),(6,4),(5,7)$ and $(7,5)$ contains all boxes $(m, n)$ with $m, n \geq 42$, but does not contain $(41,41)$. The original Putnam problem allows packings that do not have lines of cleavage; however, the corresponding rectangles are not in the Klarner system. When considering these more complex packings, Narayan and Schwenk [18] show that all $m \times n$ rectangles with $m, n \geq 34$ can be packed by $4 \times 6$ and $5 \times 7$ rectangles, but a $33 \times 33$ square cannot be packed. In fact, most of their analysis is the consideration of more complex packings.

In this example, every box satisfies the (vacuous) necessary condition to be in the Klarner system. However, there are infinitely many such boxes that are not in the Klarner system, for example, $(3, n)$ for all $n$.

\section{Asymptotically optimal box packing theorems}

Let $\mathcal{T}$ be a protoset of translation-only $d$-dimensional polyominoes.

Definition 4.1 A prime of $\mathcal{T}$ is a $b_{1} \times \cdots \times b_{d}$ box such that $\left(b_{1}, \ldots, b_{d}\right)$ is a prime of the Klarner system $\left\{\left(a_{1}, \ldots, a_{d}\right) \in \mathbb{N}^{d} \mid \mathcal{T}\right.$ packs an $a_{1} \times \cdots \times a_{d}$ box $\}$.

Thus a prime is a box that can be packed by $\mathcal{T}$, but cannot be split into two smaller boxes, both of which can be packed by $\mathcal{T}$. The reader should be aware that our definition differs slightly from Klarner and Göbel's. A prime box is strongly prime if it cannot be split into any number of smaller boxes, each of which can be packed by $\mathcal{T}$. 
This corresponds to Klarner and Göbel's definition of "prime". The distinction between these two concepts is discussed briefly in [22].

In the examples we consider below, the protoset will be invariant under rotation and/or reflection. In such cases, it is reasonable to consider two prime boxes to be "the same" if one is a rotation of the other, i.e. the dimensions of one are a permutation of the dimensions of the other.

For a protoset $\mathcal{T}$, we would like to know all boxes that can be packed by $\mathcal{T}$. If we cannot determine this, we may still ask for all sufficiently large boxes that can be packed by $\mathcal{T}$. If we can resolve this, then we have what we call an "asymptotically optimal box-packing theorem". In the special case that all the prototiles are boxes themselves, a simple calculation, as in Example 3.9 above, determines sufficient conditions for a box to be packable. Moreover, these conditions are also necessary, by Theorem 3.6, so it is easy to give an asymptotically optimal box-packing theorem in this special situation.

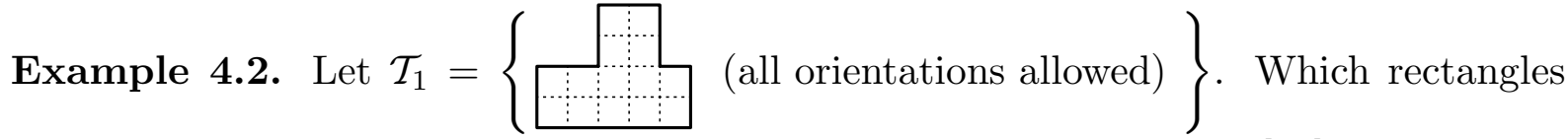
can $\mathcal{T}_{1}$ pack? It is not obvious that $\mathcal{T}_{1}$ packs any rectangle, but in [20] a packing of a $42 \times 230$ rectangle was given. We have since determined the smallest rectangle that $\mathcal{T}_{1}$ packs; it has dimensions $63 \times 80$, which answers part of Question 2 in [20]. $\mathcal{T}_{1}$ does not pack any rectangle of width less than 42 . By using a computer program based on [20, Prop. 2.1], we have succeeded in determining which rectangles of width 42 can be packed by $\mathcal{T}_{1}$, which is equivalent to finding the primes of $\mathcal{T}_{1}$ of the form $42 \times \ell$. There are 179 such primes; the shortest is $42 \times 230$ and the longest is $42 \times 3535$. $\mathcal{T}_{1}$ does not pack a $42 \times \ell$ rectangle for $\ell=3305$, but does so for all larger $\ell$. $\mathcal{T}_{1}$ also packs all $42 \times \ell$ rectangles where $\ell \geq 1852$ is even. By contrast, the shortest $42 \times \ell$ with odd $\ell$ that can be packed by $\mathcal{T}_{1}$ is $42 \times 3121$.

The extreme complexity of these packings suggests that it is unlikely we can answer the original question about determining all rectangles that $\mathcal{T}_{1}$ can pack. Nonetheless, we can determine all sufficiently large rectangles that $\mathcal{T}_{1}$ can pack.

Theorem 4.3. There is a constant $C$ such that if $m, n \geq C$, then $\mathcal{T}_{1}$ packs an $m \times n$ rectangle if and only if 14 divides $\mathrm{mn}$.

ProOF. The condition $14 \mid m n$ is clearly necessary, since the area any rectangle packed by $\mathcal{T}_{1}$ must be a multiple of 14 . We have found packings of $42 \times 230,42 \times 3121,56 \times 94$ and $63 \times 80$ rectangles. Let $S$ be the Klarner system generated by $(42,230),(230,42)$, $(42,3121),(3121,42),(56,94),(94,56),(63,80)$ and $(80,63)$. A simple calculation based on Theorem 3.8 shows that $S$ is characterized by the restrictions $\{[2,2],[7,7]\}$. These two restrictions are equivalent to the conditions $2 \mid m n$ and $7 \mid m n$, which proves the theorem.

We can also show that $C=3306$ suffices in the theorem. This is based upon packings of $42 \times n$ rectangles for all $n \geq 3306,56 \times(94+7 n)$ rectangles for all $n \geq 0$, and packings of $(63+7 m) \times 80$ rectangles for all $m \geq 0$. The best possible value of $C$ is probably much smaller. 
Example 4.4. Let $\mathcal{T}_{2}=\left\{\begin{array}{ll|lll} & & \vdots & & \\ \hline & & \vdots & \vdots & \vdots\end{array}\right.$ (all orientations allowed) $\}$. Which rectangles can $\mathcal{T}_{2}$ pack? A packing of a $28 \times 132$ rectangle is given in [20], and several necessary conditions are proved for the dimensions of a rectangle that can be packed, but they are strictly weaker than Theorem 4.5 below. Several other rectangles that can be packed by $\mathcal{T}_{2}$ are $26 \times 216,27 \times 368$ and $27 \times 376$, and we have also determined the smallest rectangle that can be packed, which has dimensions $45 \times 48$. Using these rectangles (in both orientations) and applying Theorem 3.8 , we find that $\mathcal{T}_{2}$ packs all sufficiently large rectangles that satisfy the restrictions $\{[3,3],[4,8],[8,4]\}$.

To give an asymptotically optimal box-packing theorem, we must show that these restrictions are indeed satisfied by every rectangle that can be packed by $\mathcal{T}_{2}$. We do this now.

Theorem 4.5. There is a constant $C$ such that if $m, n \geq C$, then $\mathcal{T}_{2}$ packs an $m \times n$ rectangle if and only if

(a) 3 divides $m n$, and

(b) either 4 divides $m$ or 8 divides $n$, and

(c) either 8 divides $m$ or 4 divides $n$.

ProOF. We have just seen that conditions (a), (b) and (c) are sufficient, so it remains to show that every rectangle that can be packed by $\mathcal{T}_{2}$ satisfies these conditions. Condition (a) is clearly satisfied, because the area of any rectangle packed by $\mathcal{T}_{2}$ must be divisible by 3 .

Now we show that the restriction $[4,8]$ is satisfied. If $\mathcal{T}_{2}$ packs an $m \times n$ rectangle, then it also packs a $k m \times \ell n$ rectangle for all $k, \ell>0$. If, in addition, $4 \nmid m$ and $8 \nmid n$, then $k$ and $\ell$ can be chosen so that $k m \equiv 2 \bmod 4$ and $\ell n \equiv 4 \bmod 8$. Therefore it suffices to show that $\mathcal{T}_{2}$ cannot pack a rectangle with dimensions $(4 a+2) \times(8 b+4)$. Number the squares of the infinite lattice by

$$
(i, j) \mapsto \begin{cases}1 & \text { if } i \text { and } j \text { are both even, and } i+j \equiv 0 \bmod 4, \\ 0 & \text { otherwise. }\end{cases}
$$

We note that a $2 \times 4$ rectangle placed on the infinite lattice (in either orientation) always

covers a total of 1 . The disconnected shape

always covers a total of 0 or 2 . Since the tile in $\mathcal{T}_{2}$ can be decomposed into one $2 \times 4$ rectangle, and two of the latter shape, it always covers an odd total, no matter where it is placed on the grid. On the other hand, a $(4 a+2) \times(8 b+4)$ is composed of an odd number of $2 \times 4$ rectangles, so it covers an odd total. If it could be packed by $\mathcal{T}_{2}$, the packing would use and even number of tiles, since the area is divisible by 8 . However this would mean it covers an even total, a contradiction.

This shows that the restriction $[4,8]$ is satisfied, and similarly, the restriction $[8,4]$ is also satisfied.

The above approach outlines a general approach for determining all sufficiently large boxes that can be packed by a protoset, $\mathcal{T}$, of $d$-dimensional polyominoes. On even 
numbered days, we search for box packings, and each time we find one, we adjoin it to our Klarner system, and calculate the restrictions characterizing the Klarner system. On odd numbered days, we try to prove restrictions that all boxes packed by $\mathcal{T}$ must satisfy. If, at some point, the two sets of restrictions agree, then we have an asymptotically optimal box-packing theorem.

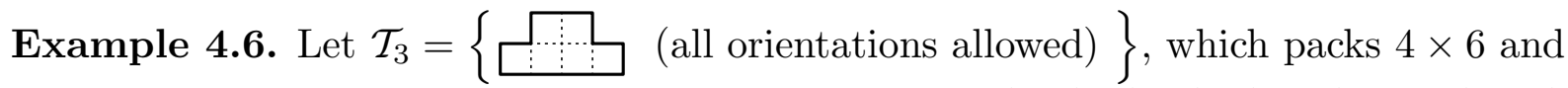
$5 \times 12$ rectangles. The Klarner system generated by $(4,6),(6,4),(5,12)$ and $(12,5)$ is characterized by the restrictions $\{[4,4],[6,6],[2,12],[12,2]\}$. Any rectangle packed by $\mathcal{T}_{3}$ satisfies the restrictions $[4,4]$ and $[6,6]$; this is proved in [22]. The restriction $[2,12]$ is equivalent to the two restrictions $[2,3]$ and $[2,4]$, which divide $[6,6]$ and $[4,4]$ respectively. Thus the restriction $[2,12]$ is implied by the two restrictions $[4,4]$ and $[6,6]$, although it is not implied by either of them individually. The same holds for $[12,2]$, so the Klarner system is characterized by $\{[4,4],[6,6]\}$. In fact, the Klarner system was determined completely in [22] in the form of giving the prime rectangles of $\mathcal{T}_{3}$, which are precisely $4 \times 6$ and $5 \times 12$.

Example 4.7. Let $\mathcal{T}_{4}=\left\{\begin{array}{llll} & \ldots & \vdots \\ \hdashline & \vdots & \vdots\end{array}\right]$ (all orientations allowed) $\}$, which has an easy packing of a $6 \times 6$ square. We have also found packings of $18 \times 25$ and $27 \times 93$ rectangles, among others. The Klarner system generated by these rectangles (and their rotations) is characterized by $\{[3,18],[18,3]\}$.

Theorem 4.8. There is a constant $C$ such that if $m, n \geq C$, then $\mathcal{T}_{4}$ packs an $m \times n$ rectangle if and only if

(a) either 3 divides $m$ or 18 divides $n$, and

(b) either 18 divides $m$ or 3 divides $n$.

PROOF. We must prove that every rectangle packed by $\mathcal{T}_{4}$ satisfies the restriction $[3,18]$; the proof for $[18,3]$ is similar. The restriction $[3,18]$ is equivalent to the two restrictions $[3,2]$ and $[3,9]$. The latter restriction is clearly satisfied because any rectangle packed by $\mathcal{T}_{4}$ must have area which is a multiple of 9 . To prove the restriction $[3,2]$ is satisfied, number the squares of the grid by $(i, j) \mapsto(-1)^{j}$. Then any placement of the tile covers a total of \pm 3 ; in particular, it covers a multiple of 3 . If $n$ is odd, then a placement of an $m \times n$ rectangle, with the side of length $m$ parallel to the $x$-axis, covers a total of $\pm m$. If the rectangle can be packed by $\mathcal{T}_{4}$, the total covered must be a multiple of 3 , so $3 \mid \mathrm{m}$. This shows that the restriction $[3,2]$ is satisfied, which completes the proof.

Example 4.9. Let $\mathcal{T}_{5}=\left\{\begin{array}{lll}\cdots & \\ \hdashline \vdots & \vdots\end{array}\right.$ (all orientations allowed) $\}$. We have found packings of $22 \times 80,22 \times 88,26 \times 64,28 \times 60$ and $36 \times 40$ rectangles, among others. Note that the $36 \times 40$ rectangle is smaller than the $32 \times 48$ rectangle, which is claimed to be minimal in [16]. The Klarner system generated by these rectangles (in all orientations) is characterized by $\{[2,0],[0,2],[4,8],[8,4]\}$.

Theorem 4.10. There is a constant $C$ such that if $m, n \geq C$, then $\mathcal{T}_{5}$ packs an $m \times n$ rectangle if and only if 
(a) $m$ and $n$ are both even, and

(b) either 4 divides $m$ or 8 divides $n$, and

(c) either 8 divides $m$ or 4 divides $n$.

Proof. If we color the squares of the lattice in a checkerboard pattern, then any placement of the tile covers 3 squares of one color and 5 of the other color. Therefore this octomino satisfies Klarner's criterion [12, Thm. 2] and hence is "even" in his terminology. This means that any rectangle packed by this shape uses an even number of tiles, so its area is divisible by 16 . This proves that the restrictions $[4,8]$ and $[8,4]$ are satisfied.

To show that each side of a rectangle packed by $\mathcal{T}_{5}$ has even length, we claim that every tile covers an even number of squares of the second row from the edge. There are four essentially different possibilities for a tile to cover an odd number of squares of that row, as shown.
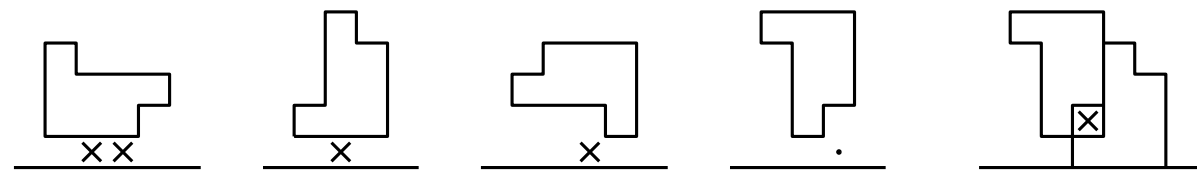

FiguRE 4.11. No tile can cover an odd number of squares of the second row.

In three of these cases, some squares are blocked so they cannot be filled. In the last case, there is only one way to fill the indicated square, and that creates a hole that cannot be filled. This proves the claim, which shows that the restrictions $[2,0]$ and $[0,2]$ are satisfied.

Example 4.12. Let $\mathcal{T}_{6}=\{\square, \square$, (all orientations allowed) $\}$, which packs $4 \times 6,5 \times 12$ and $15 \times 21$ rectangles, among others. (The first two of these rectangles use only the second prototile.) These rectangles generate all sufficiently large rectangles that satisfy the restrictions $[3,6]$ and $[6,3]$. That the restriction $[3,6]$ is satisfied by any rectangle packed by $\mathcal{T}_{6}$ was stated (without proof) in [21, Thm. 7.9]. After proving this result, we obtain an asymptotically optimal theorem.

Theorem 4.13. There is a constant $C$ such that if $m, n \geq C$, then $\mathcal{T}_{6}$ packs an $m \times n$ rectangle if and only if

(a) either 3 divides $m$ or 6 divides $n$, and

(b) either 6 divides $m$ or 3 divides $n$.

Proof. The packings of $4 \times 6,5 \times 12$ and $15 \times 21$ rectangles, used in all orientations, generate all sufficiently large rectangles that satisfy the restrictions $[3,6]$ and $[6,3]$. Now we must show that any rectangle packed by $\mathcal{T}_{6}$ satisfies these restrictions. We use the boundary word method, in the form of a representation proof, as described in [21]. Let $x=(1,2)(3,4,5)$ and $y=(1,2,3)(4,5)$ be permutations in the symmetric group $S_{5}$. We easily check that the boundary words of each orientation of each prototile is trivial in $S_{5}$, that is, $x^{2} y x^{-1} y^{2} x^{-2} y^{-1} x y^{-2}, y^{-2} x y x^{2} y^{2} x^{-1} y^{-1} x^{-2}, x^{2} y^{2} x y x^{-2} y^{-2} x^{-1} y^{-1}$, $y^{-2} x^{2} y^{-1} x y^{2} x^{-2} y x^{-1}, x^{4} y x^{-1} y x^{-2} y^{-1} x^{-1} y^{-1}, y^{-4} x y x y^{2} x^{-1} y x^{-1}, x^{-4} y^{-1} x y^{-1} x^{2} y x y$ and $y^{4} x^{-1} y^{-1} x^{-1} y^{-2} x y^{-1} x$ are all equal to the identity of $S_{5}$. If an $m \times n$ rectangle can 
be packed, then its boundary word must also be trivial, which means that $x^{m}$ commutes with $y^{n}$. Now $x$ and $y$ each have order 6 , so it is easy to check this condition. The only values $0 \leq m, n<6$ for which $x^{m}$ commutes with $y^{n}$, are $m=0, n$ arbitrary; $n=0, m$ arbitrary, and $(m, n)=(3,3)$, and in each of these cases, either 3 divides $m$ or 6 divides $n$. (In fact, this representation also shows that the restriction $[6,3]$ is satisfied.) This proves the Theorem.

In this case, we have even found all primes of $\mathcal{T}_{6}$. These are $4 \times 6,5 \times 12,10 \times 78$, $11 \times 30,13 \times 42,14 \times 18,15 \times 18,15 \times 21,15 \times 27,17 \times 18$ and $21 \times 21$. The best value of $C$ in this case is $C=16$.

We give here several more examples, where in each case, the only restrictions arise from the size of the tile dividing the area of a rectangle. (These packings, as well as others mentioned in the paper, are available from the author.)

Example 4.14. Let $\mathcal{T}_{7}=\left\{\begin{array}{l}a \\ \hdashline\end{array}\right.$ (all orientations allowed) $\}$. The smallest rectangle that can be packed by $\mathcal{T}_{7}$ has dimensions $19 \times 28$, and was found by Dahlke [5] and Marlow [15]. We have also found a packing of a $21 \times 51$ rectangle, see [20, Figure 11], and many others. From the $19 \times 28$ and $21 \times 51$ rectangles, we deduce that $\mathcal{T}_{7}$ can pack all sufficiently large rectangles whose area is a multiple of 7 .

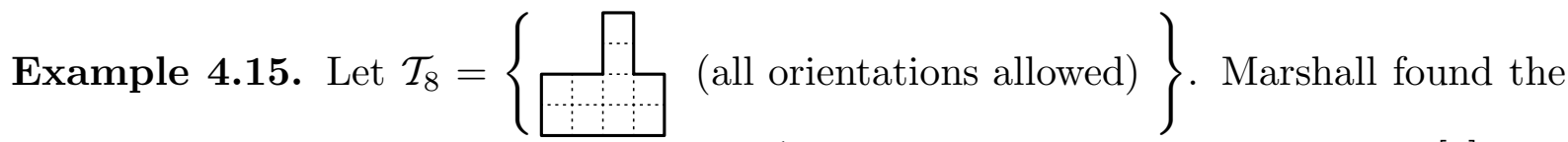
smallest rectangle that can be packed by $\mathcal{T}_{8}$, which has dimensions $30 \times 32$; see [8]. We have found packings of $18 \times 60,18 \times 65,20 \times 54$ and $20 \times 59$ rectangles, among others. These rectangles generate all sufficiently large rectangles whose area is divisible by 10 .

Example 4.16. Let $\mathcal{T}_{9}=\left\{\begin{array}{lll}\hline & & \\ \hdashline & \vdots & \vdots \\ \hdashline & : & \vdots\end{array}\right]$ (all orientations allowed) $\}$. Marshall [16] found the smallest rectangle that can be packed by $\mathcal{T}_{9}$, which has dimensions $30 \times 46$. We have found packings of $30 \times 83$ and $34 \times 65$ rectangles, as well as others. These rectangles generate all sufficiently large rectangles whose area is a multiple of 10 .

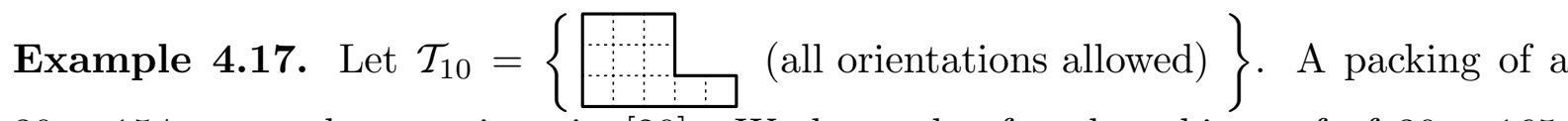
$30 \times 154$ rectangle was given in [20]. We have also found packings of of $30 \times 165$, $36 \times 187,39 \times 132,39 \times 143,41 \times 132,41 \times 143$ rectangles, as well as the smallest rectangle that can be packed, which has dimensions $54 \times 55$. These rectangles generate all sufficiently large rectangles whose area is divisible by 11 .

We conclude this section with a 3-dimensional example.

Example 4.18. Let $\mathcal{T}_{11}=\left\{\begin{array}{c}0 \\ \vdots\end{array}\right.$ shape is derived from the 2-dimensional prototile in $\mathcal{T}_{3}$, by extruding it 1 unit in the third dimension. Thus $\mathcal{T}_{11}$ has packings of $1 \times 4 \times 6$ and $1 \times 5 \times 12$ boxes obtained by extruding the packings of rectangles in the third dimension. We have also found 
packings of $3 \times 4 \times 4$ and $3 \times 5 \times 30$ boxes, as well as several others. We can now prove an asymptotically optimal theorem for this protoset.

Theorem 4.19. There is a constant $C$ such that if $m, n, p \geq C$, then $\mathcal{T}_{11}$ packs an $m \times n \times p$ box if and only if

(a) one of $m, n, p$ is a multiple of 3 , and

(b) either 2 divides $m$, or 2 divides $n$ or 6 divides $p$, and

(c) either 2 divides $m$, or 6 divides $n$ or 2 divides $p$, and

(d) either 6 divides $m$, or 2 divides $n$ or 2 divides $p$.

Proof. The $1 \times 4 \times 6,1 \times 5 \times 12,3 \times 4 \times 4$, and $3 \times 5 \times 30$ boxes above, used in all orientations, generate all sufficiently large boxes that satisfy the restrictions $[3,3,3]$, $[2,2,6],[2,6,2]$ and $[6,2,2]$.

Next we must show that these restrictions are satisfied by every box that can be packed by $\mathcal{T}_{11}$. The restriction $[3,3,3]$ is clearly satisfied, as it simply means that the volume of the box is divisible by 3 . We now prove that the restriction $[2,2,6]$ is satisfied (the proofs for $[2,2,6]$ and $[2,6,2]$ are similar). Number the cubes of the lattice by

$$
(i, j, k) \mapsto\left\{\begin{array}{cl}
(-1)^{i+j+k} & \text { if } k \text { is divisible by } 3, \\
0 & \text { otherwise. }
\end{array}\right.
$$

The prototile in $\mathcal{T}_{11}$ can be decomposed into two pieces; a $1 \times 2 \times 2$ block, and a disconnected shape of two unit cubes, three units apart. It is easy to show that any placement of either of these components on the grid covers a total of 0 , and therefore the same holds for any placement of the prototile. We also note that any placement of a $1 \times 1 \times 6$ block on the grid covers a total of 0 (this can be deduced by decomposing it into three of the disconnected shapes).

Now suppose that $m$ and $n$ are both odd, and $p$ is not divisible by 6 . An $m \times n \times p$ box can be decomposed into an $m \times n \times 6 k$ and an $m \times n \times t$ box for some $k \geq 0$ and $0<t<6$. The first box always covers a total of 0 , and it is easy to show that the second can be placed so it covers a total of \pm 1 . To achieve the latter, position the $m \times n \times t$ box with the edge of length $t$ parallel to the $z$-axis, and so that it intersects exactly one level of $(i, j, k)$ cubes with $3 \mid k$. Since $m n$ is odd, it covers a total of \pm 1 . Therefore, the $m \times n \times p$ box covers a non-zero total, so cannot be packed. This shows that the restriction $[2,2,6]$ is satisfied, which completes the proof.

\section{Some philosophy}

In the 2-dimensional examples we have considered, which includes considerably more than we have given in the previous section, the restrictions we have found are generally of three different types:

(i) restrictions that come from tile homology, i.e. from checkerboard-type arguments,

(ii) restrictions that arise from tile homotopy, i.e. from boundary word conditions (see $[4]$ ), and 
(iii) restrictions that come from "geometric" considerations.

Although there is some overlap between the types, the proofs of these restrictions seem to be sharply distinguished. For example, Theorems 4.5 and 4.8 above are proved by tile homology methods, as are the restrictions $[4,8]$ and $[8,4]$ for protoset $\mathcal{T}_{5}$, which is proved via Klarner's criterion [12, Thm. 2]. The restriction [6,6] for protoset $\mathcal{T}_{3}$, which is proved in [22], also arises from tile homology, as do all restrictions coming from considering area. The restriction $[4,4]$ for protoset $\mathcal{T}_{3}$ is proved in [22] using tile homotopy, as is Theorem 4.13 above.

It seems reasonable to believe that any restrictions that do not arise from tile homology or tile homotopy will be "geometric" in nature. Unfortunately, we are unable to give a precise definition to the notion of "geometric" in this context, so we cannot make a precise conjecture. We give some examples that hopefully convey the meaning of "geometric" restrictions. The restrictions $[2,0]$ and $[0,2]$ for protoset $\mathcal{T}_{5}$ above are proved by a geometric argument. Another example is the following.

Example 5.1. Let $\mathcal{T}_{12}=\left\{\begin{array}{llll}\hline \ldots & & \\ \hdashline & \vdots & \vdots & \vdots \\ \hdashline & & \vdots & \vdots\end{array}\right]$ (all orientations allowed) $\}$. By examining the way that $\mathcal{T}_{12}$ can fill the corner of a quadrant, we find that it can fill the first 35 diagonals, but cannot fill 36 diagonals. Therefore it cannot tessellate a quadrant, and hence cannot pack any rectangle. The relevant Klarner system is characterized by the single restriction $[0,0]$.

Hochberg and Reid [9] show that the "notched cube" in 3 dimensions and higher cannot pack any box. They prove this by showing there is a unique packing of an orthant, and the proof considers the geometry of various configurations.

Walkup [24] shows that if the T tetromino packs a rectangle, then both sides are multiples of 4 . His proof is geometric; he shows that certain patterns must propagate along diagonals in any packing of a quadrant. See also [21] where it is shown that Walkup's result cannot be obtained by tile homotopy.

The next example provides one of the more interesting geometric arguments we have encountered.

Example 5.2. Let $\mathcal{T}_{13}=\{\square, \ldots, 1, \ldots$ (all orientations allowed) $\}$. It is easy to show that the tile homotopy group of this protoset is trivial, so it does not give us any information at all. Nevertheless, this protoset has some restrictions.

Theorem 5.3. If $\mathcal{T}_{13}$ packs an $m \times n$ rectangle, then $m n$ is even.

Proof. We will show that the pentominoes (tiles with 5 squares) occur in pairs. If a pentomino occurs in the packing, the square in the gap must be filled. There are essentially two different ways to fill that square. In the first, it is filled with a pentomino, and these two are paired up. In the second case, the square is filled by a hexomino, which then creates another gap. There are two ways to fill this gap; one with a pentomino, the other with a hexomino. In the first case, the two pentominoes are paired up; 
in the second case, there is another gap created that must be filled, and the pattern continues. Thus we see that pentominoes occur in pairs, with a (possibly empty) chain of hexominoes between them.
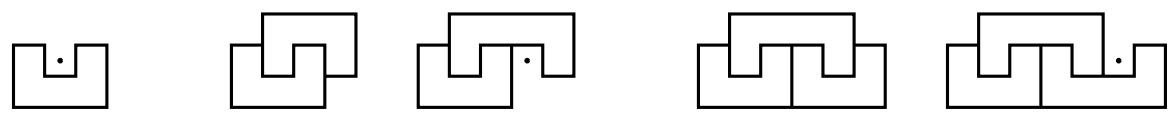

FiguRE 5.4. The pentominoes must occur in pairs.

This shows that the area of a rectangle packed by $\mathcal{T}_{13}$ must be even.

We have also found all prime rectangles for the protoset $\mathcal{T}_{13}$. There are $177 \mathrm{such}$, the smallest of which is $12 \times 14$. $\mathcal{T}_{13}$ packs all $m \times n$ rectangles where $m n$ is even and $m, n \geq 26$. Moreover, the value of 26 cannot be reduced.

To test our theory that any restrictions that do not arise from tile homology or tile homotopy must come from geometry, we will propose a specific conjecture.

Example 5.5. Let $\mathcal{T}_{14}=\left\{\begin{array}{l}\cdots \vdots \\ \hdashline \vdots \vdots\end{array}\right.$ let $\mathcal{T}_{15}=\left\{\begin{array}{l}\ldots \\ \hdashline \vdots \vdots\end{array} \ldots\right.$ (all rotations allowed, but no reflections) $\}$, where the tile has $n$ squares.

Theorem 5.6. (a) If $n$ is odd, then the tile homotopy group of $\mathcal{T}_{14}$ is cyclic of order $n$. (b) If $n$ is odd, then the tile homotopy group of $\mathcal{T}_{15}$ is cyclic of order $n$.

ProOF. (a) Recall that the tile path group is the group generated by $x$ and $y$, whose relators are boundary words of tiles. The two oriented tiles

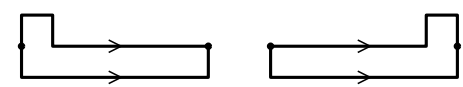

give the relations $y^{-1} x^{n-1} y=y x y^{-1} x^{n-2}$ and $y^{-1} x^{n-1} y=x^{n-2} y x y^{-1}$ in the tile path group. By equating these, we obtain $x^{n-2}\left(y x y^{-1}\right)=\left(y x y^{-1}\right) x^{n-2}$, so that $x^{n-2}$ commutes with $y x y^{-1} x^{-1}$. The two oriented tiles

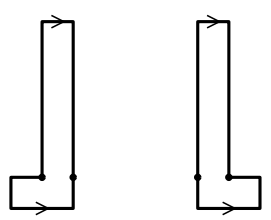

give the relations $y^{n-2} x y^{2-n}=x^{-1} y^{-1} x^{2} y$ and $y^{n-2} x y^{2-n}=y^{-1} x^{2} y x^{-1}$, so then we have $x^{2}\left(y x y^{-1} x^{-1}\right)=\left(y x y^{-1} x^{-1}\right) x^{2}$. Therefore $x^{2}$ commutes with $y x y^{-1} x^{-1}$. Because $x^{n-2}$ also commutes with this element, and $n$ is odd, it follows that $x$ commutes with $x y x^{-1} y^{-1}=\left(y x y^{-1} x^{-1}\right)^{-1}$. Since $\mathcal{T}_{14}$ is invariant under rotation, [21, Thm. 6.1] shows that its tile homotopy group is cyclic and its order is the greatest common divisor of the sizes of its prototiles. This proves (a). 
(b) $\mathcal{T}_{15}$ packs a $2 \times n$ rectangle, and therefore an $(n-1) \times n$ rectangle. By "untiling" a single tile as shown, we see that the boundary word of an $(n-1) \times(n-1)$ square with one corner removed is trivial. We can then consider this shape as a "virtual" prototile. Untile this shape from an $n \times(n-1)$ rectangle to obtain the original prototile in a reflected orientation.
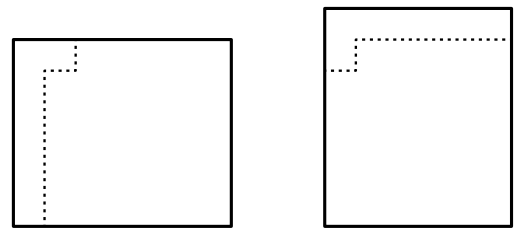

This means that in the tile path group of $\mathcal{T}_{15}$, the boundary words of the reflected tiles are all trivial. Therefore it has the same tile path group as $\mathcal{T}_{14}$, so the result now follows from (a).

It is also interesting to know what happens if $n$ is even. In the case of $\mathcal{T}_{14}$, if $n$ is divisible by 4 , then its tile homotopy group is $\mathbb{Z} \times(\mathbb{Z} / n \mathbb{Z})$, and if $n \equiv 2 \bmod 4$, then its tile homotopy group is $\mathbb{Z} \times(\mathbb{Z} / m \mathbb{Z})$, where $m=n / 2$. I do not know the general answer for $\mathcal{T}_{15}$. For $n=4$, it is $\mathbb{Z} \times(\mathbb{Z} / 4 \mathbb{Z})$ (so it is the same as for $\mathcal{T}_{14}$ ), and when $n=6$, it is $\mathbb{Z} \times(\mathbb{Z} / 15 \mathbb{Z})$. I do not know about larger even values of $n$, nor do I know if the tile homotopy group is abelian in these cases.

Theorem 5.6 says that, for odd $n$, the tile homotopy groups of $\mathcal{T}_{14}$ and $\mathcal{T}_{15}$ can only detect area modulo $n$. Since we do not expect there to be any "geometric" restrictions, we expect that to be the only necessary condition for large rectangles to be packable.

Conjecture 5.7. Suppose $n$ is odd.

(a) There is a constant $C$ such that $\mathcal{T}_{14}$ packs all $a \times b$ rectangles, when $a, b \geq C$ and $n$ divides $a b$.

(b) There is a constant $C$ such that $\mathcal{T}_{15}$ packs all $a \times b$ rectangles, when $a, b \geq C$ and $n$ divides $a b$.

When $n$ is odd, $\mathcal{T}_{14}$ packs an $(n+2) \times 3 n$ rectangle; see $[16,20]$. If $n$ is prime, this is sufficient to deduce part (a) of the conjecture, with $C=n+1$. We have also found enough packings of rectangles to confirm part (a) when $n=9$. Part (b) of the conjecture is stronger; it implies part (a). We have also confirmed part (b) for $n=5$ and $n=7$. (For $n=3$, the two protosets coincide, so both parts of the conjecture are true.) For $n=5$, we have even found all the prime rectangles; they are $2 \times 5,13 \times 55$, $15 \times 39,17 \times 35$ and $19 \times 25$.

The conjecture, if true, would provide an infinite family of examples that support our philosophy, which would carry more weight than individual examples. This is our main reason for interest.

It is an intriguing question if there is a higher dimensional analogue of the ConwayLagarias boundary word method. We might hope that a higher dimensional analogue of our philosophy should hold (although we do not have enough evidence yet). If tiling 
restrictions can be classified into different types as in the 2-dimensional case, it is likely that this will provide some insight into this important question.

\section{References}

[1] F.W. Barnes, Algebraic Theory of Brick Packing II, Discrete Mathematics 42 (1982), no. 2-3, 129-144. MR 84e:05044b

[2] Olivier Bodini, Tiling a Rectangle with Polyominoes, Discrete Models for Complex Systems, DMCS'03 (Michel Morvan and Éric Rémila, eds.), 2003, pp. 81-88.

[3] Richard A. Brualdi and Thomas H. Foregger, Packing Boxes with Harmonic Bricks, Journal of Combinatorial Theory, Series B 17 (1974), no. 2, 81-114. MR 53 \#5342

[4] J.H. Conway and J.C. Lagarias, Tiling with Polyominoes and Combinatorial Group Theory, Journal of Combinatorial Theory, Series A 53 (1990), no. 2, 183-208. MR 91a:05030

[5] Karl A. Dahlke, A Heptomino of Order 76, Journal of Combinatorial Theory, Series A 51 (1989), no. 1, 127-128. MR 90b:05042

Erratum, Journal of Combinatorial Theory, Series A 52 (1990), no. 2, 321. MR 90j:05053

[6] N.G. de Bruijn and D.A. Klarner, A Finite Basis Theorem for Packing Boxes with Bricks, Philips Research Reports 30 (1975), 337-343.

[7] Solomon W. Golomb, Tiling with Polyominoes, Journal of Combinatorial Theory 1 (1966), no. 2, 280-296. MR 33 \#6498

[8] Solomon W. Golomb, Tiling Rectangles with Polyominoes, The Mathematical Intelligencer 18 (1996), no. 2, 38-47. MR 97f:05047

[9] Robert Hochberg and Michael Reid, Tiling with Notched Cubes, Discrete Mathematics 214 (2000), no. 1-3, 255-261. MR 2001a:52022

[10] G. Katona and D. Szász, Matching Problems, Journal of Combinatorial Theory, Series B 10 (1971), 60-92. MR 46 \#798

[11] D.A. Klarner and F. Göbel, Packing Boxes with Congruent Figures, Indagationes Mathematicae 31 (1969), 465-472. MR 40 \#6362

[12] David A. Klarner, Packing a Rectangle with Congruent N-ominoes, Journal of Combinatorial Theory 7 (1969), no. 2, 107-115. MR 40 \#1894

[13] David A. Klarner, A Finite Basis Theorem Revisited, Technical Report STAN-CS73-338, Stanford University (February 1973), 10 pp. (unpublished) http://wwwdb.stanford.edu/TR/CS-TR-73-338.html

[14] Earl S. Kramer, Tiling Rectangles with $T$ and $C$ Pentominoes, Journal of Recreational Mathematics, 16 (1983-84), no. 2, 102-113.

[15] T.W. Marlow, Grid Dissections, Chessics 23 (1985), 78-79.

[16] William Rex Marshall, Packing Rectangles with Congruent Polyominoes, Journal of Combinatorial Theory, Series A 77 (1997), no. 2, 181-192. MR 98e:05026 
[17] Cristopher Moore and John Michael Robson, Hard Tiling Problems with Simple Tiles, Discrete \& Computational Geometry 26 (2001), no. 4, 573-590. MR 2002h:52029

[18] Darren A. Narayan and Allen J. Schwenk, Tiling Large Rectangles, Mathematics Magazine 75 (2002), no. 5, 372-380.

[19] J. L. Ramírez Alfonsín, The Frobenius Diophantine Problem, Oxford Lecture Series in Mathematics and its Applications, 30, Oxford University Press, Oxford, 2005. MR 2007i:11052

[20] Michael Reid, Tiling Rectangles and Half Strips with Congruent Polyominoes, Journal of Combinatorial Theory, Series A 80 (1997), no. 1, 106-123. MR 98i:05046

[21] Michael Reid, Tile Homotopy Groups, L'Enseignement Mathématique 49 (2003), no. 1-2, 123-155. MR 2004f:52020

[22] Michael Reid, Klarner Systems and Tiling Boxes with Polyominoes, Journal of Combinatorial Theory, Series A, 111 (2005), no. 1, 89-105. MR 2006a:52018

[23] Stan Wagon, Fourteen Proofs of a Result about Tiling a Rectangle, American Mathematical Monthly 94 (1987), no. 7, 601-617. MR 89h:52018

[24] D.W. Walkup, Covering a Rectangle with T-tetrominoes, American Mathematical Monthly 72 (1965), 986-988. MR 32 \#1582 